Pacific Journal of Mathematics

A PHRAGMÉN-LINDELÖF THEOREM FOR FUNCTION

IRVING LEONARD GLICKSBER 


\section{A PHRAGMEN-LINDELÖF THEOREM FOR FUNCTION ALGEBRAS}

\section{GLICKSBERG}

Let $A$ be a function algebra, considered as a closed subalgebra of $C(\mathfrak{M})$, where $\mathfrak{M}$ is the space of multiplicative linear functionals on $A$. Let $\partial$ denote the Šilov boundary of $A$. We shall call $\mathfrak{M} \backslash \partial$ the "interior of $\mathfrak{M}$ " and say a function $g$ on this "interior" is $A$-holomorphic if each $\varphi$ in $\mathfrak{M} \backslash \partial$ has a neighborhood on which $g$ is uniformly approximable by elements of $A$.

What we shall observe here is that results of the Phragmén-Lindelöf type apply to certain $A$-holomorphic functions.

These results follow easily from the type of argument used in an earlier paper [1] in which function-algebra analogues of some classical results of function theory were obtained; the present note is essentially an addendum to [1] (where " $A$-holomorphic" [3] was "locally approximable"). Other results of the Phragmén-Lindelöf type have been obtained by Quigley [2].

Our analogue of the usual Phragmén-Lindelöf result replaces the point at infinity by a peak set lying in the Silov boundary.

THEOREM 1. Suppose $f \in A$ peaks on $F \subset \partial$, and $g$ is an A-holomorphic function defined and continuous on $\mathfrak{M} \backslash F$. Suppose $g$ is bounded on $0 \backslash F$ and for some $\alpha, 0<\alpha<1$, and $k>0$

$$
g \exp \left(\frac{-k}{|1-f|^{\alpha}}\right)
$$

is bounded on the interior of $\mathfrak{M}$. Then $g$ is bounded on $\mathfrak{M} \backslash F$ by its bound on $\partial \backslash F$.

Thus an unbounded $A$-holomorphic function continuous on $\mathfrak{M} \backslash F$ cannot increase too slowly as we approach $F$. Actually $g$ need only be defined on $\mathfrak{M} \backslash \partial$ (and $A$-holomorphic) if we replace $\partial \backslash F$ by a deleted neighborhood of it in $\mathfrak{M}$.

THEOREM 2. With $f, F$ and $\alpha$ as above, let $g$ be an A-holomorphic function which is bounded on the intersection $V$ of a neighborhood of $\partial \backslash F$ with the interior of $\mathfrak{M}$, and suppose (1) holds. Then $g$ is bounded by its bound on $V$.

Both of these results are easy consequences of the local maximum 
modulus principle [4] and classical arguments. A little more is needed for the following extension of the Phragmén-Lindelöf corollary concerning a bounded analytic function on a sector having a limit as $z \rightarrow \infty$ along the bounding rays.

THEOREM 3. Suppose $g$ is a bounded function on $\mathfrak{M}$ which is A-holomorphic, has its restriction to $\partial$ continuous, and in fact is continuous at each point of $\partial \backslash \bigcup_{n=1}^{\infty} K_{n}$, where $K_{n}$ is a zero set of $A$ lying in the ${ }^{(1)}$ Choquet boundary. Then $g$ is continuous on $\mathfrak{M}$.

Thus we cannot have too small a set of discontinuities for an $A$-holomorphic function which has a continuous restriction to the Šilov boundary and also is continuous at a fairly large set of points in $\partial$.

As a mixture of Theorems 1 and 3 we obtain

Corollary 4. Suppose $g$ is a (not necessarily bounded) function on $\mathfrak{M}$ which is A-holomorphic, has its restriction to $\partial$ continuous and is continuous at each point of $\partial \backslash \bigcup_{n=1}^{\infty} K_{n}$, where $K_{n}$ is a zero set of A lying in the Choquet boundary. Suppose $f \in A$ peaks on $K_{1}$ while (1) is bounded on the interior of $\mathfrak{M}$. Then $g$ is continuous on Mi.

Proofs. Our proof of Theorem 1 is simply an imitation of a classical argument [5]. To begin let $\alpha<\beta<1$; noting that

$$
|\arg (1-f)| \leqq \pi / 2 \text {, }
$$

we have an element $(1-f)^{\beta}$ in $A$ (where we apply the principal branch of $z^{\beta}$ to $1-f$, so $\left.\left|\arg (1-f)^{\beta}\right| \leqq \beta \pi / 2<\pi / 2\right)$. Now fix $\beta$ and $\varepsilon>0$. For $\operatorname{Re} z \geqq 0$ and $z=\mathrm{re}^{i \theta} \neq 0(|\theta| \leqq \pi / 2)$

$$
\begin{aligned}
\left|\exp \left(-\frac{\varepsilon}{z^{\beta}}\right) \exp \frac{k}{|z|^{\alpha}}\right| & =\exp \left(-\varepsilon r^{-\beta} \cos \beta \theta+k r^{-\alpha}\right) \\
& =\exp \left\{-r^{-\beta}\left(\varepsilon \cos \beta \theta-k r^{\beta-\alpha}\right)\right\} \\
& \leqq \exp \left(-c r^{-\beta}\right)
\end{aligned}
$$

for some $c>0$ if $r$ is sufficiently small, and this of course implies (2) is bounded on $\operatorname{Re} z \geqq 0$. Thus

(1) The Choquet boundary consists of all points in the Šlov boundary having unique representing measures. In the metric case it coincides with the set of peak points. 


$$
\exp \left(-\frac{\varepsilon}{(1-f)^{\beta}}\right) \exp \left(\frac{k}{|1-f|^{\alpha}}\right)
$$

is bounded on $\mathfrak{M} \backslash F$, whence

$$
g \exp \left(-\frac{\varepsilon}{(1-f)^{3}}\right)
$$

is bounded on $\mathfrak{M} \backslash F$ as the product of (1) and (3). But the exponential in (4), and thus (4) itself, is $A$-holomorphic and we can argue that by [1, Th, 4.8], (4) is bounded on $\mathfrak{M} \backslash F$ by its bound over $\partial \backslash F$, hence by $\sup |g(\partial \backslash F)|$ since the exponential is of modulus $\leqq 1$. So for any $\varphi$ in $\mathfrak{M} \backslash F$ we have

$$
\left.\left|g(\phi) \exp \left(-\frac{\varepsilon}{(1-f(\varphi))^{\beta}}\right)\right| \leqq \sup \mid g(\partial) \backslash F\right) \mid,
$$

and letting $\varepsilon \rightarrow 0$ yields the desired result.

Actually, once we have seen (4) is a bounded $A$-holomorphic function we should appeal directly to Rossi's local maximum modulus principle [4] to obtain (5). Indeed, extend (4) to all of $\mathfrak{M}$ by setting it equal to zero on $F$; since (3) tends to zero as we approach $F$ (by (2)) we obtain a continuous function $h$ on $\mathfrak{M}$. Now let $B$ be the closed subalgebra of $C(\mathfrak{M})$ generated by $h$ and $A$. To obtain (5) we need only see $\partial_{B} \subset \partial$ since then

$$
|h(\varphi)| \leqq \sup \left|h\left(\partial_{B}\right)\right| \leqq \sup |h(\partial)|=\sup |h(\partial \backslash F)|,
$$

because $h$ vanishes on $F$, and this is (5).

We now argue exactly as in $[1,3.2]$ : if $\phi \in \partial_{B} \cap(\mathfrak{M} \mid \partial)$ we choose a neighborhood $\boldsymbol{U}_{\varphi}$ of $\varphi$ in $\mathfrak{M} \mid \partial$ on which $h$ (and thus any element of $B$ ) is uniformly approximable by elements of $A$. Since $\varphi \in \partial_{B}$ we must have a $\varphi^{\prime}$ in $\boldsymbol{U}_{\varphi}$ and an $h^{\prime}$ in $B$ with

$$
\left|h^{\prime}\left(\varphi^{\prime}\right)\right|>\sup \mid h^{\prime}\left(\text { bndry } \boldsymbol{U}_{\varphi}\right) \mid
$$

and thus this holds for some approximating element $h^{\prime \prime}$ in $A$. But that violates the local maximum modulus principle, so $\partial_{B} \cap(\mathfrak{M} \mid \partial)=\varnothing$, and $\partial_{B} \subset \partial$.

This argument yields a simple proof of Theorem 2 . In that result, as is now apparent, we need only show the function

$$
h=g \exp \left(\frac{-\varepsilon}{(1-f)^{\beta}}\right)
$$

on $\mathfrak{M} \mid \partial$ is bounded by its bound on $V$.

Now choose a deleted neighborhood $W$ of $F$ on which 


$$
|h|<\sup |h(V)|+\eta
$$

(where $\eta>0$ ), which is possible since $h \rightarrow 0$ as we approach $F$, exactly as before. Removing $(V \cup W)^{-}$from the interior $\mathfrak{M} \mid \partial$ we obtain an open subset $U$ of $\mathfrak{M} \backslash \partial$ with $U^{-} \cap \partial=\varnothing$ so that bndry $U \subset(V \cup W)^{-}$. With $B$ now the closed subalgebra of $C\left(U^{-}\right)$generated by $A$ and $h$ we see that $\partial_{B} \subset$ bndry $U$ by just the above application of local maximum modulus. Hence $\partial_{B} \subset(V \cup W)^{-}$, so that

$$
\sup |h(\mathfrak{M} \backslash \partial)| \leqq \sup |h(V \cup W)| \leqq \sup |h(V)|+\eta ;
$$

since $\eta>0$ is arbitrary, this shows $h$ is bounded by its bound over $\boldsymbol{V}$, as desired.

We can now proceed to the proof of Theorem 3, which involves some modifications in the arguments of $[1, \S 4]$. Let $B_{0}$ denote the uniformly closed algebra of bounded functions on $\mathfrak{M}$ generated by $g$ and $A$; trivially $\mathfrak{M}$ can be viewed as a subset of $\mathfrak{M}_{B_{0}}$ and we let $X$ denote the closure of $\mathfrak{M}$ in $\mathfrak{M}_{B_{0}}$. $X$ is a boundary for $B_{0}$, so $B=B_{0}^{\wedge} \mid X$ is a closed subalgebra of $C(X)$.

Since $g$ and the elements of $A$ are continuous when restricted to either $\partial$ or $\mathfrak{M} \mid \partial$, the natural injection of each of these spaces into $X$ is continuous, and of course one-to-one. In particular then the compact space $\partial$ is imbedded homeomorphically in $X$. But in fact the same is true of $\mathfrak{M} \mid \partial$ since the map $\rho: X \rightarrow \mathfrak{M}$ dual to $A \rightarrow B$ clearly provides inverses for the injections $\partial \rightarrow X$, Ml $\mid \partial \rightarrow X$. (Note that $\hat{f}(x)=$ $f(\rho(x))$ for $f \in A, x \in X$.)

Now each of the sets $\mathfrak{M} \backslash \partial$ and $\partial \backslash(\mathfrak{M} \mid \partial)^{-}$is imbedded as an open subset of $X$. To see this note that each $\varphi_{0}$ in $\left.\mathfrak{M}\right\rangle \partial$ has a compact neighborhood in $\mathfrak{M}$ disjoint from $\partial$ of the form

$$
U=\left\{\phi \in \mathfrak{M}_{:}:\left|f_{i}(\varphi)-\mathrm{f}_{i}\left(\varphi_{0}\right)\right| \leqq \varepsilon, \quad i=1, \cdots, n\right\} ;
$$

since $X=(\mathfrak{M} \backslash U)^{-} \cup U^{-}=(\mathfrak{M} \backslash U)^{-} \cup U, x \in X \backslash U$ implies $\quad x \in(\mathfrak{M} \backslash U)^{-}$, and so $\left|\hat{f}_{i}(x)-f_{i}\left(\varphi_{0}\right)\right| \geqq \varepsilon$ for some $i$, whence

$$
\begin{aligned}
W_{\varphi_{0}} & =\left\{\phi \in \mathfrak{M}_{i}:\left|f_{i}(\varphi)-f_{i}\left(\varphi_{0}\right)\right|<\varepsilon / 2, i=1, \cdots, n\right\} \\
& =\left\{x \in X:\left|\hat{f}_{i}(x)-f_{i}\left(\varphi_{0}\right)\right|<\varepsilon / 2, i=1, \cdots, n\right\}
\end{aligned}
$$

is a neighborhood of $\varphi_{0}$ in $X$ lying wholly within $\mathfrak{M} \backslash \partial$, so $\mathfrak{M} \mid \partial$ is open in $X$ as asserted. The same argument, starting from a compact neighborhood in $\partial$ disjoint from $(\mathfrak{M} \mid \partial)^{-}$, yields a neighborhood $W_{\varphi_{0}}$ of $\varphi_{0} \in \partial \backslash(\mathfrak{M} \backslash \partial)^{-}$in $X$ lying wholly in $\partial \backslash(\mathfrak{M} \backslash \partial)^{-}$, so this set is also open in $X$. Moreover, the existence of $W_{\varphi_{0}}$ shows $\rho$ is one-to-one over $\mathfrak{M} \backslash \partial$ and $\partial \backslash(\mathfrak{M} \backslash \partial)^{-}$. For $\hat{f}_{i}(x)=\hat{f}_{i}(\rho(x)), x \in X, f_{i} \in A$, so $\rho(x) \in \mathfrak{M} \backslash \partial \partial$ implies $x \in W_{p(x)} \subset \mathfrak{M} \backslash \partial$; similarly $\rho(x) \in \partial \backslash(\mathfrak{M} \ell \mid \partial)^{-}$implies $x \in \partial \backslash(\mathfrak{M} \backslash \partial)^{-}$. So

$$
\rho^{-1}(\mathfrak{M} \backslash \partial)=\mathfrak{M} \backslash \partial, \rho^{-1}\left(\partial \backslash(\mathfrak{M} \backslash \partial)^{-}\right)=\partial \backslash(\mathfrak{M} \backslash \partial)^{-},
$$


and thus $\rho$ is clearly one-to-one over these sets.

Since $\mathfrak{M} \backslash \partial$ is open in $X$ local maximum modulus applies to show $\partial_{B} \cap(\mathfrak{M} \backslash \partial)=\varnothing$ exactly as in $[1,3.2]$ or in our proof of Theorem 1: for any $\varphi \in \partial_{B} \cap(\mathfrak{M} \backslash \partial)$ has a neighborhood $U_{\varphi}$ in $\mathfrak{M} \backslash \partial \partial$ on which $g$ (and so any element of $B$ ) is uniformly approximable by elements of $A$; since $\mathfrak{M} \backslash \partial$ is open in $X, U_{\varphi}$ is open in $X$ and thus we find $\varphi^{\prime}$ in $U_{\varphi}$ and $h^{\prime}$ in $B$ satisfying (6) since $\varphi \in \partial_{B}$, and this contradicts local maximum modulus exactly as in the proof of Theorem 1 . Thus

$$
\partial_{B} \cap(\mathfrak{M} \backslash \partial)=\varnothing,
$$

and since $\rho^{-1}(\mathfrak{M} \backslash \mid \partial)=\mathfrak{M} \backslash \partial$, we conclude that $\rho\left(\partial_{B}\right) \subset \partial$.

To complete our proof we need only see $\rho$ is one-to-one on $X$ : for then $\rho$ is a homeomorphism of $X$ with $\mathfrak{M}$ (since $\rho(X) \subset \mathfrak{M}$ and $\left.\rho(X) \supset(\mathfrak{M} \backslash \partial) \cup\left(\partial \backslash(\mathfrak{M} \backslash \partial)^{-}\right)\right)$, and continuity of $g \circ \rho=\hat{g}$ on $X$ implies that of $g$ on $\mathfrak{M}=\rho(X)$.

We have already seen $\rho^{-1}(x)=\{x\}$ for $x$ in $(\mathfrak{M} \backslash \partial) \cup\left(\partial \mid(\mathfrak{M} \mid \partial)^{-}\right)$, and for $x$ in $\partial \backslash \bigcup K_{n}$ the assumed continuity of $g$ at $x$ implies $\rho^{-1}(x)=\{x\}$ : for each $h$ in $B_{0}$ is continuous at $x$, and so if $\rho(y)=x$ and the net $\left\{\varphi_{\delta}\right\}$ in $\mathfrak{M}$ converges to $y$ in $X$ then $\rho\left(\varphi_{\delta}\right)=\varphi_{\delta} \rightarrow \rho(y)=x$ in $\mathfrak{M}$, whence $\hat{h}(y)=\lim \hat{h}\left(\varphi_{\delta}\right)=\hat{h}(x)$ for all $h$ in $B_{0}$, and $y=x$. Thus we need only see $\rho(y)=x$ for $x$ in $K_{n}$ implies $y=x$, and since we know this holds for $x$ in $\partial \backslash(\mathfrak{M} \backslash \partial)^{-}$, we can assume $x \in(\mathfrak{M} \backslash \partial)^{-}$as well.

So suppose $\rho(y)=x \in K_{n} \cap(\mathfrak{M} \backslash \partial)^{-}$. Since $K_{n}$ lies in the Choquet boundary of $A$, only the unit point mass $\delta_{x}$ at $x$, among all probability measures on $\partial$, can represent $x$ on $A$. Thus if we knew $\partial_{B}=\partial$ then any probability measure $\mu$ on $\partial_{B}=\partial$ representing $y$ on $B$ would necessarily represent $\rho(y)=x$ on $A$, whence $\mu=\delta_{x}$ and $y=x$.

So we need only see $\partial_{B} \mid \partial=\varnothing$ (since clearly $\partial \subset \partial_{B}$ ). As we saw, $\rho\left(\partial_{B}\right) \subset \partial$, and $\rho$ is one-to-one over $\left(\partial \backslash \bigcup K_{n}\right) \cup\left(\partial \backslash\left(\mathfrak{M} \backslash\langle\partial)^{-}\right)\right.$so that

$$
\rho\left(\partial_{B} \backslash \partial\right) \subset(\mathfrak{M} \backslash \partial)^{-} \cap\left(\mathbf{U} K_{n}\right) .
$$

So by category if $\partial_{B} \mid \partial \neq \phi$ one of the closed sets

$$
E_{n}=\rho^{-1}\left[K_{n} \cap(\mathfrak{M} \mid \partial)^{-}\right] \cap\left(\partial_{B} \mid \partial\right)
$$

in the locally compact space $\partial_{B} \backslash \partial$ has nonvoid interior in $\partial_{B} \backslash \partial$, hence in $\partial_{B}$. But $K_{n}=g_{n}^{-1}(0), g_{n} \in A$ so that $y \in E_{n}$ lies in $\hat{g}_{n}^{-1}(0)=\left(g_{n} \circ \rho\right)^{-1}(0)$. In fact $y$ lies in the topological boundary in $X$ of $\hat{g}_{n}^{-1}(0)$. For

$$
\rho(y) \in(\mathfrak{M} \backslash \partial)^{-}, y \notin \partial,
$$

and thus $y$ has a neighborhood in $X$ disjoint from $\partial$, whence $y$ lies in the closure in $X$ of $\mathfrak{M} \backslash \partial$ (since $(\mathfrak{M} \backslash \partial) \cup \partial$ is dense in $X$ ). But $\hat{g}_{n}^{-1}(0) \cap(\mathfrak{M} \backslash \partial)=g_{n}^{-1}(0) \cap(\mathfrak{M} \backslash \partial)=\phi$, so that $y$ lies in the topological 
boundary of $\hat{g}_{n}^{-1}(0)$ as asserted.

Thus we have seen that $E_{n}$ has nonvoid interior in $\partial_{B}$ and lies in the topological boundary of $\hat{g}_{n}^{-1}(0)$ in $X$, which contradicts [1, 2.2]. Our assumption that $\partial_{B} \mid \partial$ is nonvoid must therefore be false, and $\partial_{B}=\partial$ as desired, completing our proof.

Corollary 4 follows directly from the preceding. Indeed if we set

$$
h=\left\{\begin{array}{lll}
g \exp \left(\frac{-\varepsilon}{(1-f)^{\beta}}\right) & \text { on } \mathfrak{M} \backslash K_{1} & \\
0 & \text { on } K_{1}, \quad \alpha<\beta<1,
\end{array}\right.
$$

then $h \mid \partial$ is continuous and Theorem 3 implies $h \in C(\mathfrak{M})$. So $h$ is bounded by its bound over $\partial$, exactly as in the proof of Theorem 1 , and so we see the same is true of $g$. Hence by Theorem $3, g \in C(\mathfrak{M})$.

\section{REFERENCES}

1. I. Glicksberg, Maximal algebras and a theorem of Radó, Pacific. J. Math. 14 (1964), 919-941; correction, 19 (1966), 587.

2. Frank Quigley, Generalized Phragmen-Lindelöf Theorems, Function Algebras (Proc. Intenat. Sympos., Tulane Univ., 1965), 36-41. Scott-Foresman, Chicago, 1966.

3. C. E. Rickart, Analytic phenomena in general function algebras, Pacific J. Math. 18 (1966), 361-377.

4. H. Rossi, The local maximum modulus principle, Ann. of Math. 72 (1960), 1-11.

5. E. C. Titchmarsh, The Theory of Functions, London, 1932.

Received April 19, 1966. Work supported in part by the National Science Foundation.

UNIVERSITY OF WASHINGTON 


\section{PACIFIC JOURNAL OF MATHEMATICS}

\section{EDITORS}

\section{H. SAMELSON}

Stanford University

Stanford, California

J. P. JANS

University of Washington

Seattle, Washington 98105
J. DUGUNDJI

University of Southern California Los Angeles, California 90007

RICHARD ARENS

University of California

Los Angeles, California 90024

\section{ASSOCIATE EDITORS}
E. F. BECKENBACH
B. H. NEumanN
F. WOLF
K. YosidA

\section{SUPPORTING INSTITUTIONS}

\author{
UNIVERSITY OF BRITISH COLUMBIA \\ CALIFORNIA INSTITUTE OF TECHNOLOGY \\ UNIVERSITY OF CALIFORNIA \\ MONTANA STATE UNIVERSITY \\ UNIVERSITY OF NEVADA \\ NEW MEXICO STATE UNIVERSITY \\ OREGON STATE UNIVERSITY \\ UNIVERSITY OF OREGON \\ OSAKA UNIVERSITY \\ UNIVERSITY OF SOUTHERN CALIFORNIA
}

\author{
STANFORD UNIVERSITY \\ UNIVERSITY OF TOKYO \\ UNIVERSITY OF UTAH \\ WASHINGTON STATE UNIVERSITY \\ UNIVERSITY OF WASHINGTON \\ AMERICAN MATHEMATICAL SOCIETY \\ CHEVRON RESEARCH CORPORATION \\ TRW SYSTEMS \\ NAVAL ORDNANCE TEST STATION
}

Mathematical papers intended for publication in the Pacific Journal of Mathematics should be typewritten (double spaced). The first paragraph or two must be capable of being used separately as a synopsis of the entire paper. It should not contain references to the bibliography. Manuscripts may be sent to any one of the four editors. All other communications to the editors should be addressed to the managing editor, Richard Arens at the University of California, Los Angeles, California 90024.

50 reprints per author of each article are furnished free of charge; additional copies may be obtained at cost in multiples of 50 .

The Pacific Journal of Mathematics is published monthly. Effective with Volume 16 the price per volume ( 3 numbers) is $\$ 8.00$; single issues, $\$ 3.00$. Special price for current issues to individual faculty members of supporting institutions and to individual members of the American Mathematical Society: $\$ 4.00$ per volume; single issues $\$ 1.50$. Back numbers are available.

Subscriptions, orders for back numbers, and changes of address should be sent to Pacific Journal of Mathematics, 103 Highland Boulevard, Berkeley 8, California.

Printed at Kokusai Bunken Insatsusha (International Academic Printing Co., Ltd.), 7-17, Fujimi 2-chome, Chiyoda-ku, Tokyo, Japan.

PUBLISHED BY PACIFIC JOURNAL OF MATHEMATICS, A NON-PROFIT CORPORATION

The Supporting Institutions listed above contribute to the cost of publication of this Journal, but they are not owners or publishers and have no responsibility for its content or policies. 


\section{Pacific Journal of Mathematics

Vol. 22, No. $3 \quad$ March, 1967

Wai-Mee Ching and James Sai-Wing Wong, Multipliers and $H^{*}$

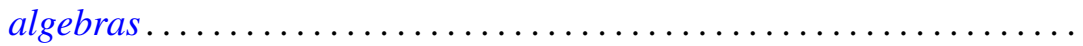

P. H. Doyle, III and John Gilbert Hocking, A generalization of the Wilder

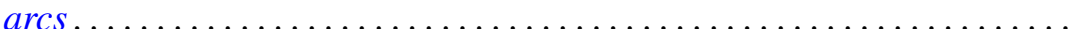

Irving Leonard Glicksberg, A Phragmén-Lindelöf theorem for function

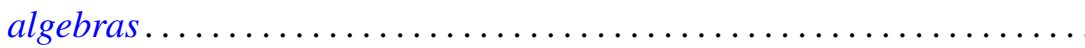

E. M. Horadam, A sum of a certain divisor function for arithmetical semi-groups ..................................... 407

V. Istrăţescu, On some hyponormal operators ................... 413

Harold H. Johnson, The non-invariance of hyperbolicity in partial

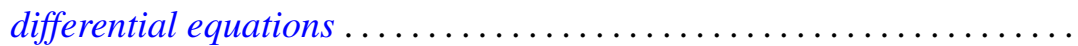

Daniel Paul Maki, On constructing distribution functions: A bounded denumerable spectrum with $n$ limit points................... 431

Ronald John Nunke, On the structure of Tor. II .................... 453

T. V. Panchapagesan, Unitary operators in Banach spaces ............. 465

Gerald H. Ryder, Boundary value problems for a class of nonlinear differential equations ................................. 477

Stephen Simons, The iterated limit condition and sequential convergence .................................... 505

Larry Eugene Snyder, Stolz angle convergence in metric spaces ......... 515 Sherman K. Stein, Factoring by subsets ................... 523

Ponnaluri Suryanarayana, The higher order differentiability of solutions of abstract evolution equations . . .

Leroy J. Warren and Henry Gilbert Bray, On the square-freeness of Fermat and Mersenne numbers ............................... 563

Tudor Zamfirescu, On l-simplicial convexity in vector spaces........... 565 Eduardo H. Zarantonello, The closure of the numerical range contains the spectrum 